\title{
Improved capture of adverse events after spinal surgery procedures with a longitudinal administrative database
}

\author{
*Anand Veeravagu, MD, Tyler S. Cole, BS, Tej D. Azad, BA, and John K. Ratliff, MD \\ Department of Neurosurgery, Stanford University School of Medicine, Palo Alto, California
}

OBJECT The significant medical and economic tolls of spinal disorders, increasing volume of spine surgeries, and focus on quality metrics have made it imperative to understand postoperative complications. This study demonstrates the utility of a longitudinal administrative database for capturing overall and procedure-specific complication rates after various spine surgery procedures.

METHODS The Thomson Reuters MarketScan Commercial Claims and Encounters and the Medicare Supplemental and Coordination of Benefits database was used to conduct a retrospective analysis of longitudinal administrative data from a sample of approximately 189,000 patients. Overall and procedure-specific complication rates at 5 time points ranging from immediately postoperatively (index) to 30 days postoperatively were computed.

RESULTS The results indicated that the frequency of individual complication types increased at different rates. The overall complication rate including all spine surgeries was $13.6 \%$ at the index time point and increased to $22.8 \%$ at 30 days postoperatively. The frequencies of wound dehiscence, infection, and other wound complications exhibited large increases between 10 and 20 days postoperatively, while complication rates for new chronic pain, delirium, and dysrhythmia increased more gradually over the 30-day period studied. When specific surgical procedures were considered, 30 -day complication rates ranged from $8.6 \%$ in single-level anterior cervical fusions to $27.3 \%$ in multilevel combined anterior and posterior lumbar spine fusions.

CONCLUSIONS This study demonstrates the usefulness of a longitudinal administrative database in assessing postoperative complication rates after spine surgery. Use of this database gave results that were comparable to those in prospective studies and superior to those obtained with nonlongitudinal administrative databases. Longitudinal administrative data may improve the understanding of overall and procedure-specific complication rates after spine surgery.

http://thejns.org/doi/abs/10.3171/2014.12.SPINE14659

KEY WORDS spine surgery; outcomes research; postoperative complications; administrative database; longitudinal; spinal disorders

$\mathrm{T}$ HE medical and economic burdens of spine disorders pose a growing challenge for patients, medical providers, and society. In 2005, 33.3 million US adults $(15 \%)$ had spinal ailments whose treatment and other expenditures resulted in an estimated total cost of $\$ 85.9$ billion. The most common International Classification of Disease, Ninth Revision, Clinical Modification (ICD-9$\mathrm{CM})$ diagnoses of these spinal disorders included "other and unspecified disorders of the back" (52.9\%), "intervertebral disc disorders" $(15.9 \%)$, and "sprains or strains of the back" $(9.3 \%)$. Expenditures for managing or treating these conditions, after adjustment for age, sex, and inflation, increased by $65 \%$ from 1997 to 2005, but health out- comes did not improve commensurate with these rising costs. ${ }^{12}$ One strategy to improve quality of spine surgical care is to gain improved and generalizable knowledge of adverse events after spine surgery to aid in their prevention and in improved patient selection.

As the number of surgical treatments for spinal disorders increase and new health care regulations emphasize specific postoperative metrics, it is critical to understand the array of complications and expected incidences that may occur after surgical intervention. Although the incidences of complications and adverse events after spine surgery are becoming more defined, a large amount of disparate findings remains that varies by surgical level and

\footnotetext{
ABBREVIATIONS CPT = Current Procedural Terminology; ICD-9-CM = International Classification of Disease, Ninth Revision, Clinical Modification; NIS = National (Nationwide) Inpatient Sample.

SUBMITTED July 8, 2014. ACCEPTED December 30, 2014

INCLUDE WHEN CITING Published online June 12, 2015; DOI: 10.3171/2014.12.SPINE14659.

DISCLOSURE This work was supported by a research grant from the Orthopedic Research and Education Foundation (Grant OREF 112-209), the Walsh Foundation, and the Stanford University Medical Scholars Program. CPT codes are a registered trademark of the American Medical Association.

* Dr. Veeravagu and Mr. Cole contributed equally to this work.
} 
study design. ${ }^{14}$ Variation in reporting and analysis both lead to an unclear understanding of the complications after spine surgery.

Administrative databases have been used extensively to assess complications associated with spine surgery. The National (Nationwide) Inpatient Sample (NIS) is a widely used database compiled from the Healthcare Cost and Utilization Project of the Agency for Healthcare Research and Quality (see http://www.hcup-us.ahrq.gov). The NIS offers patient-level data, but only for individual hospital admissions. Because of a lack of individual patient identifiers that may be tracked over time after discharge or transfer, follow-up in the NIS database is lost. Cases of readmissions cannot be captured, and cases of wound infections and other complications that may be present after discharge cannot be assessed.

Longitudinal databases offer patient-specific identifiers that may be tracked over time. ${ }^{19}$ Hence, health care claims after discharge, hospital readmissions, and adverse events developing after the time of index procedure are captured. This capture may provide a more accurate assessment of any postoperative adverse events.

Improving the understanding of procedure-specific complication rates is useful to surgeons, patients, ancillary providers, and payers. Here, we show that longitudinal databases enable capture of overall and procedure-specific complications at rates approaching those observed in prospective studies. This study also places our longitudinal data within the context of other retrospective studies of adverse events after spine surgery. Longitudinal databases provide a robust collection of patient data and offer a valuable retrospective assessment of complications occurring in the perioperative and postoperative periods of spine surgery.

\section{Methods \\ Literature Review}

We searched the National Center for Biotechnology Information PubMed database for studies analyzing complications, adverse events, and outcomes after spine surgery. We initially queried the PubMed database by using the Boolean operator "AND" with the terms "spine surgery," "adverse event," and "complication." We expanded our survey to include searches for specific events that we analyzed with longitudinal data and queried the database for procedure and complication combinations such as "lumbar fusion" AND "hematoma." We then selected studies that considered complications investigated in our present analysis. We included both prospective and retrospective studies that drew data from several different databases and patient samples. Articles in languages other than English and studies conducted to record events not analyzed in our present study were excluded. This selection resulted in 20 studies whose scope and data represented a number of complications after spine surgery, diverse databases, and different study designs.

\section{Study Design and Database}

This study has been approved by the Stanford Institutional Review Board. All patient information and follow- up data were obtained and analyzed in accordance with the Helsinki Declaration, patient privacy was protected according to the US Health Insurance Portability and Accountability Act (HIPAA), and all procedures were in compliance with an institutional review board-approved protocol.

We performed a retrospective observational administrative database study of records from patients in the United States who underwent spine surgical procedures. Inpatient and outpatient longitudinal data for this study were obtained from the Thomson Reuters MarketScan Commercial Claims and Encounters and the Medicare Supplemental and Coordination of Benefits database from 2006 through 2010. These annual data sets include data from 100 payers and comprised inpatient, outpatient, and pharmacy services from large employers, health plans, governmental, and public organizations.

\section{Cohort Selection}

We queried the MarketScan database using Current Procedural Terminology (CPT) and ICD-9-CM codes (Supplemental Table 1). In the studied period between 2006 and 2010, there were 188,965 patients who met our primary inclusion criteria.

\section{Complication Assessment}

To define the time point of complication onset, we chose the date of surgery according to the presence of a CPT code indicating an operative procedure. Patients were excluded if they were in an associated diagnosisrelated group or if their condition had an ICD-9-CM code indicating trauma or neoplastic involvement. The aggregation format in MarketScan allowed for identification of the initial, or index, hospital stay for a given procedure. To recreate the limitations of incident-only, nonlongitudinal data, in our "index" group we truncated follow-up to the initial hospitalization where the procedure in question was performed. This eliminated the potential benefits of the longitudinal data capturing complication rates associated with the procedure at follow-ups. The rates of complications at the index hospitalization were then compared with the rates at various postoperative time points. These postoperative complications were identified through readmissions, clinic visits, and other claims not registered during the patient's initial inpatient stay.

The complications were chosen according to clinical relevance and because they were most commonly assessed in the literature. To avoid attributing preexisting diagnoses as a new onset in the analysis, a postoperative ICD9-CM code in the patient's record was only considered if it was not present within a year before the operation. Complications for which this restriction applied included chronic pain, deep vein thrombosis, pulmonary embolism, dysphagia, dysrhythmia, neurological complications, and pulmonary complications.

To measure the increase in captured complication rates, time points were chosen at which to calculate the cumulative frequency of each complication within 30 days postoperatively. The index complication rate represented the incidence of a complication during the operative hospi- 
tal stay. The rate at each time point $(5,10,20$, or 30 days postoperatively) represented the percentage of the patients who had a given complication diagnosed on the basis of administrative claims data within that postoperative window. If a complication occurred before discharge, and if the length of stay was longer than a given time point, the complication was counted as occurring within the initial stay. From these rates, we calculated the percent increase relative to the index rate for each complication.

\section{Statistical Analysis}

Data were prepared and analyzed with SAS software (version 9.3; SAS Institute, Inc.).

\section{Results}

\section{Literature Review of Complication Rates in Spine Surgery}

We identified 19 studies that reported spine surgery procedures and complications comparable to those considered in the current analysis (Table 1). Studies varied by procedure performed, adverse events recorded, sample size, time point of outcomes, and type of study. Of these 20 studies, 13 had been performed retrospectively, and 13 drew on large national databases for patient data. The studies' investigators determined and presented the rates of both overall and specific complications for a range of spine surgery procedures and follow-up periods. Pulmonary $(13.0 \%)$ and hematological $(10.8 \%)$ complications were the more common adverse events in spine surgery, ${ }^{10}$ while pulmonary embolisms (1.0\%) and wound-related complications $(1.2 \%-3.2 \%)$ were the least common. ${ }^{6.7}$

In a retrospective analysis, Chitale et al. observed that lumbar spine surgery resulted in greater complication rates $(13.6 \%)$ than cervical spine surgery $(4.5 \%) .{ }^{4}$ In a prospective assessment, Yadla et al. reported a similar finding, noting that complication rates were higher (59.4\%) in operations in the thoracolumbar spine than in the cervical spine (47.1\%). ${ }^{22,23}$ Several of the prospective studies identified reported overall complication rates ranging from $44.5 \%$ to $59.4 \%$ at 30 days postoperatively. These rates were uniformly greater than overall complication rates determined by any retrospective study at 30 days postoperatively, including our data (27\%). In a prospective study by Bazaz et al., the rate of dysphagia after anterior cervical spine surgery fell greatly from $50.2 \%$ to $12.5 \%$ over the 1-year study period. ${ }^{1}$ A retrospective study by Patil et al. reported complication rates to be relatively stable or slightly increased between immediately after the operation and 90 days postoperatively. ${ }^{15}$

\section{Total Complication Rates After Spine Surgery}

We queried the MarketScan database for the 12 most common complications after spine surgery (Table 2). For a range of spinal procedures (Table 3), we determined rates of adverse events at the index time point. We also estimated cumulative complication rates at 5, 10, 20, and 30 days postoperatively to measure the increased capture of complications otherwise undetected by databases limited to the immediate inpatient setting.

Complication rates for each observed adverse event increased from the index to the 30-day time point (Table
2). However, the increases of the individual complications varied over time. Wound dehiscence, infection, and other wound complications showed large relative increases in frequency, with the steepest increases occurring between 10 and 20 days postoperatively, while complication rates for new chronic pain, delirium, and dysrhythmia increased more gradually over the 30-day postoperative period. Nonwound-related complications occurred earlier after the index surgery, whereas wound-related events were both present and developed over the course of the 30-day postoperative period. The overall complication rate for the all spine interventions analyzed here was $13.6 \%$ at the index time point and increased to $22.8 \%$ at 30 days postoperatively.

\section{Differential Increases in Complication Rate}

We identified 16 different spine procedures in the MarketScan database and calculated their complication rates at discrete time points ranging from the index time point to 30 days postoperatively (Table 3 ). This analysis indicated variation in complication rates according to surgical approach, number of levels operated on, and use of decompression. For instance, the complication rates at 30 days postoperatively ranged from $8.6 \%$ in single-level anterior cervical fusions to $27.3 \%$ in multilevel combined anterior and posterior lumbar spine fusions. Furthermore, the complications varied in the level of increase relative to the index rate, with some adverse events showing higher rates 30 days postoperatively relative to the index rate than others. Overall complication rates of the surgical procedures were also calculated to assess variability in complication rates based on procedure. The overall procedure-specific complications also varied in frequency over time.

\section{Discussion}

In this study, we demonstrate the value of a longitudinal database for capturing rates of complications arising from spine surgery. We also present these results in the context of both prospective and retrospective studies, which have investigated complications of a broad range of spinal procedures.

\section{Database Comparison}

Effective use of longitudinal databases stands to improve the quality of retrospective studies on spine surgery outcomes. The advantages of observational data over randomized controlled trial data include being able to study a more diverse patient population and observing the effects of a treatment under real-world, rather than idealized, conditions. Although randomized trials are the gold standard for medical evidence, the surgical community is increasingly recognizing that in many circumstances this "Level A" evidence is costly to generate and may not be timely. Often, study participants are narrowly selected in these trials, and the results of such studies are therefore not generalizable.

For specific demographic groups that might be underrepresented in trials, large anonymized data sets gleaned from existing electronic medical records or claims data may enable a clinician to address patient-centered ques- 


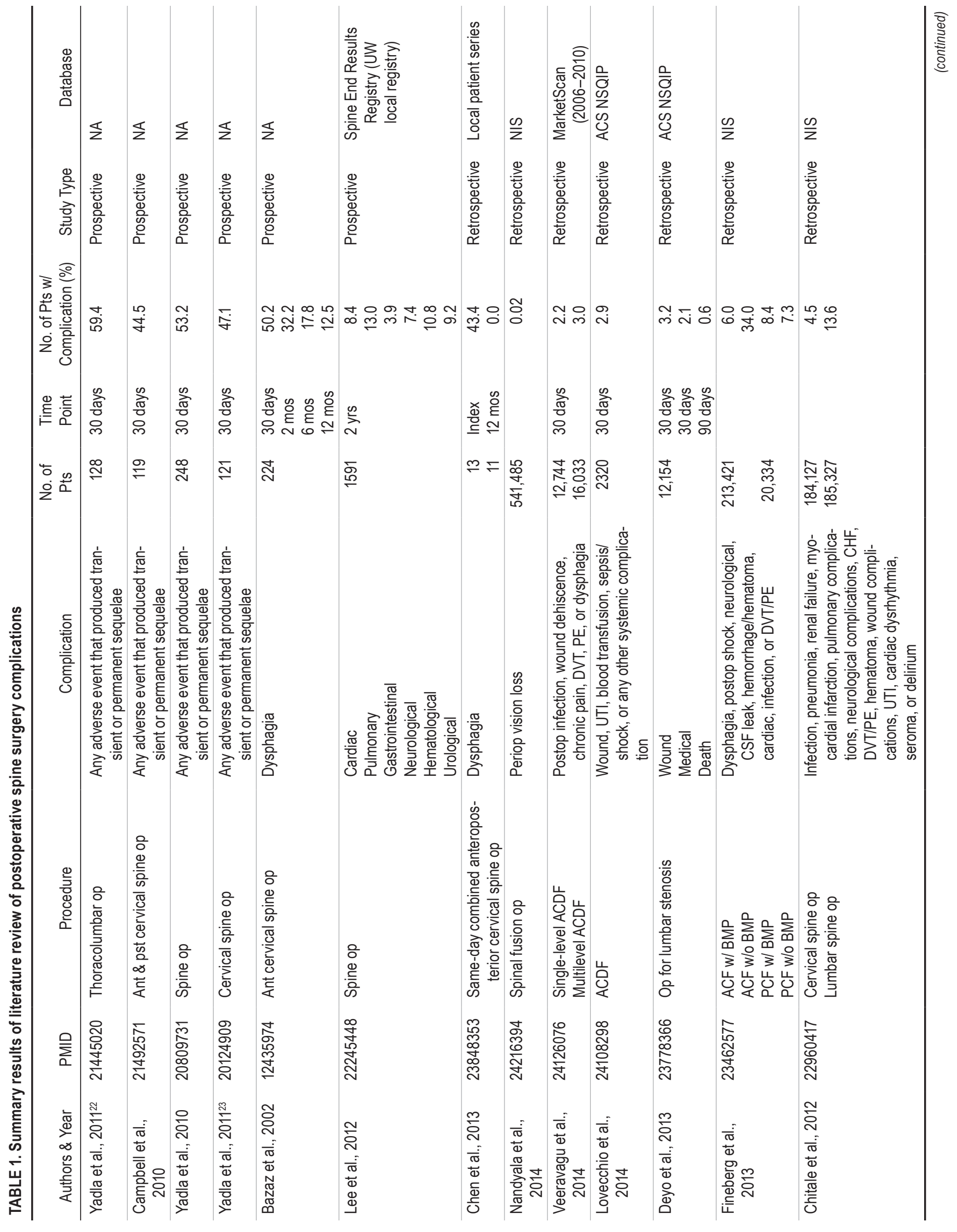




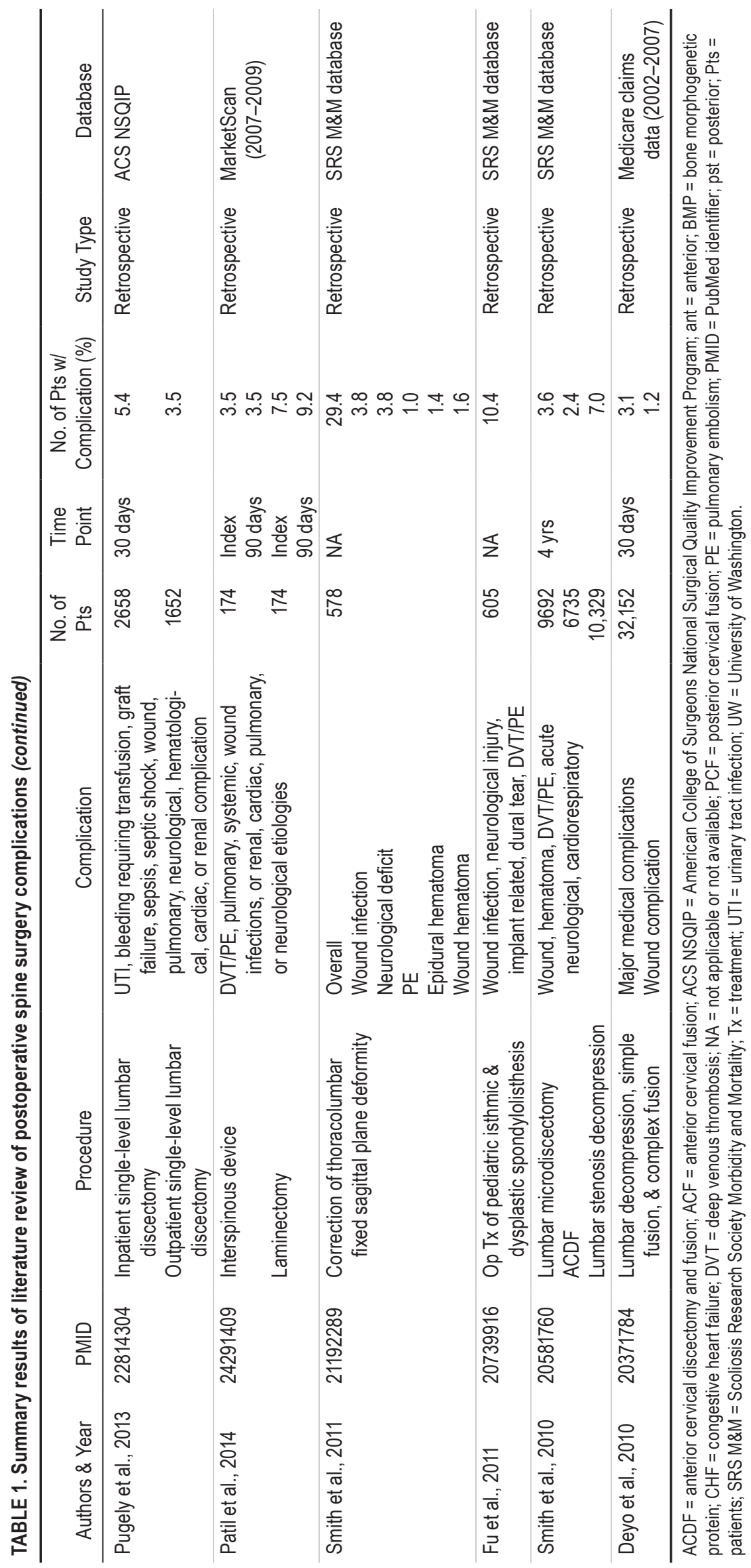


TABLE 2. Rates of postoperative spinal surgery complications immediately after the operation (index) and in the 30 -day period after discharge

\begin{tabular}{|c|c|c|c|c|c|}
\hline \multirow[b]{2}{*}{ Complication } & \multicolumn{5}{|c|}{ Complication Rate (\%) } \\
\hline & Index & 5 Days & $\begin{array}{c}10 \\
\text { Days }\end{array}$ & $\begin{array}{c}20 \\
\text { Days }\end{array}$ & $\begin{array}{c}30 \\
\text { Days }\end{array}$ \\
\hline Wound dehiscence & 0.21 & 0.25 & 0.37 & 0.66 & 0.86 \\
\hline Wound infection & 1.2 & 1.4 & 1.9 & 3.0 & 3.6 \\
\hline Wound hematoma & 1.0 & 1.1 & 1.3 & 1.6 & 1.8 \\
\hline $\begin{array}{l}\text { Other wound complica- } \\
\text { tion }\end{array}$ & 0.41 & 0.50 & 0.73 & 1.2 & 1.5 \\
\hline New chronic pain & 1.1 & 1.2 & 1.2 & 1.4 & 1.5 \\
\hline Delirium & 0.34 & 0.38 & 0.42 & 0.49 & 0.56 \\
\hline DVT & 1.5 & 1.6 & 2.0 & 2.4 & 2.8 \\
\hline PE & 0.83 & 0.88 & 0.97 & 1.1 & 1.3 \\
\hline Dysphagia & 1.2 & 1.4 & 1.5 & 1.8 & 2.1 \\
\hline Dysrhythmia & 7.4 & 7.7 & 8.1 & 8.6 & 9.1 \\
\hline $\begin{array}{l}\text { Neurological complica- } \\
\text { tion }\end{array}$ & 3.4 & 3.5 & 3.8 & 4.1 & 4.4 \\
\hline Pulmonary complication & 5.8 & 6.1 & 6.4 & 7.0 & 7.5 \\
\hline \multirow[t]{2}{*}{ All } & 13.6 & 14.0 & 17.2 & 20.0 & 22.8 \\
\hline & \multicolumn{5}{|c|}{ Increase Over Index Rate (\%) } \\
\hline Wound dehiscence & NA & 16.3 & 76.4 & 213.5 & 308.2 \\
\hline Wound infection & NA & 13.1 & 56.3 & 139.9 & 194.4 \\
\hline Wound hematoma & NA & 10.5 & 31.1 & 61.7 & 80.5 \\
\hline $\begin{array}{l}\text { Other wound complica- } \\
\text { tion }\end{array}$ & NA & 22.4 & 77.7 & 190.3 & 272.4 \\
\hline New chronic pain & NA & 5.9 & 13.2 & 27.4 & 39.2 \\
\hline Delirium & NA & 10.9 & 23.0 & 46.0 & 64.2 \\
\hline DVT & NA & 10.3 & 32.1 & 65.0 & 89.0 \\
\hline PE & NA & 5.7 & 17.0 & 35.4 & 50.9 \\
\hline Dysphagia & NA & 12.4 & 27.0 & 50.7 & 72.5 \\
\hline Dysrhythmia & NA & 4.2 & 8.5 & 16.4 & 23.0 \\
\hline $\begin{array}{l}\text { Neurological complica- } \\
\text { tion }\end{array}$ & NA & 5.1 & 11.6 & 22.0 & 30.2 \\
\hline Pulmonary complication & NA & 5.0 & 10.5 & 20.7 & 29.8 \\
\hline All & NA & 5.4 & 13.6 & 26.7 & 35.8 \\
\hline
\end{tabular}

tions in a practical manner and to study the effectiveness and risk factors of a treatment. Accordingly, our results do not represent only a single university's or a few institutions' experience; rather, our use of a longitudinal database provided a wide-angle lens of a patient population independent of institution-specific constraints.

Yet despite the utility of retrospective and longitudinal databases for studying outcomes in large patient samples, we note that clinical data are aggregated and structured in ways specific to the organization or company administrating the database. Thus, retrospective databases are not monolithic, with higher subscription fees generally relating to higher-quality clinical or patient-level data. For example, the NIS draws from a broad group of hospitals (that is, from approximately $20 \%$ of hospitals nationally), but it does not offer longitudinal patient tracking after dis- charge. In contrast, the MarketScan database has patientlevel, longitudinal tracking as well as detailed information on insurance status, while still drawing on a large nationwide hospital sample. The high level of detail at the patient level translates to a higher cost for use of the MarketScan database than that of NIS. The MarketScan database is composed primarily of private payer records; however, the Medicare Provider Utilization and Payment Data has similar patient-level, longitudinal data with similarly high user fees. In addition, the database providers may differ in how procedures are tracked, such as with ICD-9-CM codes in addition to diagnosis-related groups versus with CPT codes directly.

Other databases used retrospectively are being adapted from prospectively collected data, such as the Spine End Results Registry and the American College of Surgeons National Surgical Quality Improvement Program. The advantage of these databases is the granularity of the assessed variables, which often may be more clinically relevant and do not rely on algorithms to extract patient comorbidities from administrative data. However, these types of databases lack insurance and payer information, making it more difficult to conduct cost comparisons and address other socioeconomic questions. The Scoliosis Research Society has yet another paradigm in retrospective database construction: surgeon-reported outcomes. This specific scope has the advantage of focused clinical outcomes most relevant to surgical practitioners, yet also suffers from a lack of socioeconomic data in addition to poor delineations of patient comorbidity measures.

With focused and statistically sound leveraging of database research, randomized controlled trials may be better planned to match surgeons' most pressing questions and to clarify conflicting results identified in retrospective data assessments. If a given question is adequately addressed with retrospective data, then resources need not necessarily be dedicated to answering it with resource-intensive prospective trials. Furthermore, retrospective data may be used to determine whether the results from trials hold in practice, or to assess complications that were not initially measured and that original studies were not powered to detect. ${ }^{5,20}$

\section{Results of This Study}

Using an administrative, claims-based, longitudinal database, we found an overall complication rate of $13.6 \%$ at index hospitalization (that is, complications identified during a patient's initial admission with a billing claim for surgery) that increased to a cumulative $22.8 \%$ at the 30 day follow-up. These rates were comparable to what has been encountered in practice and closely reflected rates observed in prospective studies on spinal surgery complications. Adverse events such as wound dehiscence, infection, and other wound complications showed large relative increases during discrete postoperative time periods, while rates for complications such as new chronic pain, delirium, and dysrhythmia displayed more even and moderate increases over the observed postoperative period.

For the index time point of our study, we truncated our data analysis to the initial hospital stay where a procedure of interest was performed. By limiting our analysis to the index hospitalization only, we eliminated the potential 
TABLE 3. Postoperative complication rates by procedure at the index time point and in the 30 -day period after discharge

\begin{tabular}{|c|c|c|c|c|c|c|}
\hline \multirow[b]{2}{*}{ Procedure } & \multirow[b]{2}{*}{ No. of Pts } & \multicolumn{5}{|c|}{ Complication Rate (\%) } \\
\hline & & Index & 5 Days & 10 Days & 20 Days & 30 Days \\
\hline Ant thoracolumbar & 8178 & 14.4 & 14.9 & 16.2 & 18.4 & 19.7 \\
\hline \multicolumn{7}{|l|}{ Lumbar decompression } \\
\hline Pst & 61,591 & 8.0 & 8.5 & 9.5 & 11.1 & 12.1 \\
\hline Pst \& ant & 668 & 12.7 & 13.2 & 14.2 & 15.7 & 16.9 \\
\hline \multicolumn{7}{|l|}{ Lumbar fusion } \\
\hline Single level, pst & 7773 & 9.1 & 9.6 & 10.8 & 12.8 & 14.0 \\
\hline Single level, pst \& ant & 1472 & 11.8 & 12.4 & 13.6 & 15.9 & 17.3 \\
\hline Single level, pst, w/ decompression & 26,416 & 9.2 & 9.8 & 10.9 & 12.9 & 14.1 \\
\hline Single level, pst \& ant, w/ decompression & 1650 & 11.0 & 11.6 & 12.8 & 15.4 & 16.6 \\
\hline Multiple levels & 7447 & 16.7 & 17.3 & 18.9 & 21.5 & 23.3 \\
\hline Multiple levels, pst \& ant & 1902 & 20.5 & 21.5 & 22.9 & 25.7 & 27.3 \\
\hline Multiple levels, pst, w/ decompression & 21,991 & 13.6 & 14.5 & 16.1 & 18.6 & 20.2 \\
\hline Multiple levels, pst \& ant, w/ decompression & 1412 & 15.6 & 16.6 & 18.6 & 22.2 & 23.8 \\
\hline \multicolumn{7}{|l|}{ ACDF } \\
\hline Single level, w/ decompression & 21,672 & 5.6 & 6.4 & 7.0 & 7.8 & 8.6 \\
\hline Multiple levels, w/ decompression & 25,508 & 7.2 & 8.3 & 9.1 & 10.0 & 10.8 \\
\hline \multicolumn{7}{|l|}{ Cervical corpectomy } \\
\hline Single level & 2373 & 8.9 & 9.5 & 10.5 & 11.1 & 12.1 \\
\hline Multiple levels & 4514 & 11.6 & 12.9 & 14.1 & 15.5 & 16.5 \\
\hline Multiple levels, w/ decompression & 1168 & 12.2 & 13.7 & 14.6 & 16.0 & 17.0 \\
\hline
\end{tabular}

benefits of longitudinal patient-level data and modeled the output of the MarketScan database to parallel assessments with databases such as the NIS. As shown in Table 2, this produced a much more limited assessment of postoperative complications, with many later adverse events being missed because of this focus on only the index time point. This finding indicates that the use of longitudinal data results in potentially superior accuracy in identifying postoperative complications.

In comparing our results to those in studies investigating similar spine surgery outcomes in Table 1, we call attention to the various study types selected and data sources leveraged. Our selection included both prospective and retrospective studies, with the latter drawing on a range of database types including surgeon reported (Scoliosis Research Society Morbidity and Mortality Database), retrospective nonlongitudinal (NIS), retrospective longitudinal (National Surgical Quality Improvement Program), and longitudinal administrative (MarketScan). Overall, the prospective studies indicated higher complication rates than the retrospective studies. This was expected, as investigators actively collect patient information at regular intervals, reducing recall error.

However, the degree of symptom transiency or which recorded complications specifically correlate with patient quality of life is unclear. Despite the lower recall of complications associated with retrospective data, these data generally represent complications that are more representative of what care providers must address clinically. The MarketScan database used in the current study has the advantage of containing longitudinal administrative data across different facilities and payers; thus, any changes in location of service or insurance status do not affect the data collection. With this method of data curation, we find that administrative data can assess complications more reflective of those assessed with prospective data (such as less recall error) yet still represent data that are encountered in clinical practice and likely to represent significant patient events.

\section{Limitations}

Despite the aforementioned advantages of using longitudinal administrative data, it is important to consider the limitations of this study. First, we note that the direct comparison of complication rates from our data to the rates obtained from the literature should be viewed with caution given the different methods of data selection and analysis of the various series included. Comparisons of complication rates among surgeries were also limited because indications for the procedures and more detailed contextual information were not included in this analysis. For example, MarketScan does not provide information about which level of the spine was surgically treated or which specific levels have an increased incidence of complications.

In addition, administrative database studies rely on accurate coding of both the index procedures and the complications; coding errors may lead to inappropriate overor underreporting of events and may vary depending on treatment outcomes. Moreover, the MarketScan database used in this study primarily contained records from indi- 
viduals with private insurance, which may limit the generalizability of our results. Although we evaluated some of the most common postoperative complications, others are not well assessed with administrative data. Thus, our analysis did not include all potential complications nor did it achieve granularity beyond the ICD-9-CM definitions. As database design and content quality continue to improve, these limitations will become less significant when retrospective data are applied to answering clinical questions. Taken together, longitudinal databases stand to markedly improve our understanding of the postoperative course following spine surgery.

\section{Conclusions}

This study demonstrates that postoperative complication rates of spine surgery obtained with data from a large longitudinal administrative database are comparable to rates observed in prospective studies. Longitudinal administrative data are superior to nonlongitudinal administrative data and may contribute to an improved understanding of overall and procedure-specific complication rates after spine surgery.

\section{References}

1. Bazaz R, Lee MJ, Yoo JU: Incidence of dysphagia after anterior cervical spine surgery: a prospective study. Spine (Phila Pa 1976) 27:2453-2458, 2002

2. Campbell PG, Yadla S, Malone J, Zussman B, Maltenfort MG, Sharan AD, et al: Early complications related to approach in cervical spine surgery: single-center prospective study. World Neurosurg 74:363-368, 2010

3. Chen CJ, Saulle D, Fu KM, Smith JS, Shaffrey CI: Dysphagia following combined anterior-posterior cervical spine surgeries. J Neurosurg Spine 19:279-287, 2013

4. Chitale R, Campbell PG, Yadla S, Whitmore RG, Maltenfort MG, Ratliff JK: International Classification of Disease Clinical Modification 9 modeling of a patient comorbidity score predicts incidence of perioperative complications in a nationwide inpatient sample assessment of complications in spine surgery. J Spinal Disord Tech [epub ahead of print], 2012

5. Cole T, Veeravagu A, Jiang B, Ratliff JK: Usage of recombinant human bone morphogenetic protein in cervical spine procedures: analysis of the MarketScan longitudinal database. J Bone Joint Surg Am 96:1409-1416, 2014

6. Deyo RA, Hickam D, Duckart JP, Piedra M: Complications following surgery for lumbar stenosis in a veteran population. Spine (Phila Pa 1976) 38:1695-1702, 2013

7. Deyo RA, Mirza SK, Martin BI, Kreuter W, Goodman DC, Jarvik JG: Trends, major medical complications, and charges associated with surgery for lumbar spinal stenosis in older adults. JAMA 303:1259-1265, 2010

8. Fineberg SJ, Ahmadinia K, Oglesby M, Patel AA, Singh K: Hospital outcomes and complications of anterior and posterior cervical fusion with bone morphogenetic protein. Spine (Phila Pa 1976) 38:1304-1309, 2013

9. Fu KMG, Smith JS, Polly DW Jr, Perra JH, Sansur CA, Berven SH, et al: Morbidity and mortality in the surgical treatment of six hundred five pediatric patients with isthmic or dysplastic spondylolisthesis. Spine (Phila Pa 1976) 36:308312, 2011

10. Lee MJ, Konodi MA, Cizik AM, Bransford RJ, Bellabarba C, Chapman JR: Risk factors for medical complication after spine surgery: a multivariate analysis of 1,591 patients. Spine J 12:197-206, 2012

11. Lovecchio F, Hsu WK, Smith TR, Cybulski G, Kim B, Kim
JYS: Predictors of thirty-day readmission after anterior cervical fusion. Spine (Phila Pa 1976) 39:127-133, 2014

12. Martin BI, Deyo RA, Mirza SK, Turner JA, Comstock BA, Hollingworth W, et al: Expenditures and health status among adults with back and neck problems. JAMA 299:656-664, 2008

13. Nandyala SV, Marquez-Lara A, Fineberg SJ, Singh R, Singh $\mathrm{K}$ : Incidence and risk factors for perioperative visual loss after spinal fusion. Spine J 14:1866-1872, 2014

14. Nasser R, Yadla S, Maltenfort MG, Harrop JS, Anderson DG, Vaccaro AR, et al: Complications in spine surgery. J Neurosurg Spine 13:144-157, 2010

15. Patil CG, Sarmiento JM, Ugiliweneza B, Mukherjee D, Nuño M, Liu JC, et al: Interspinous device versus laminectomy for lumbar spinal stenosis: a comparative effectiveness study. Spine J 14:1484-1492, 2014

16. Pugely AJ, Martin CT, Gao Y, Mendoza-Lattes SA: Outpatient surgery reduces short-term complications in lumbar discectomy: an analysis of 4310 patients from the ACS-NSQIP database. Spine (Phila Pa 1976) 38:264-271, 2013

17. Smith JS, Fu KMG, Polly DW Jr, Sansur CA, Berven SH, Broadstone PA, et al: Complication rates of three common spine procedures and rates of thromboembolism following spine surgery based on 108,419 procedures: a report from the Scoliosis Research Society Morbidity and Mortality Committee. Spine (Phila Pa 1976) 35:2140-2149, 2010

18. Smith JS, Sansur CA, Donaldson WF III, Perra JH, Mudiyam R, Choma TJ, et al: Short-term morbidity and mortality associated with correction of thoracolumbar fixed sagittal plane deformity: a report from the Scoliosis Research Society Morbidity and Mortality Committee. Spine (Phila Pa 1976) 36:958-964, 2011

19. Veeravagu A, Cole T, Jiang B, Ratliff JK: Revision rates and complication incidence in single- and multilevel anterior cervical discectomy and fusion procedures: an administrative database study. Spine J 14:1125-1131, 2014

20. Veeravagu A, Cole TS, Jiang B, Ratliff JK, Gidwani RA: The use of bone morphogenetic protein in thoracolumbar spine procedures: analysis of the MarketScan longitudinal database. Spine J 14:2929-2937, 2014

21. Yadla S, Malone J, Campbell PG, Maltenfort MG, Harrop JS, Sharan AD, et al: Early complications in spine surgery and relation to preoperative diagnosis: a single-center prospective study. J Neurosurg Spine 13:360-366, 2010

22. Yadla S, Malone J, Campbell PG, Maltenfort MG, Sharan AD, Harrop JS, et al: Preoperative diagnosis and early complications in thoracolumbar spine surgery: a single center prospective study. J Spinal Disord Tech 24:E16-E20, 2011

23. Yadla S, Malone J, Campbell PG, Nasser R, Maltenfort MG, Harrop JS, et al: Incidence of early complications in cervical spine surgery and relation to preoperative diagnosis: a singlecenter prospective study. J Spinal Disord Tech 24:50-54, 2011

\section{Author Contributions}

Conception and design: all authors. Acquisition of data: Veeravagu, Cole, Azad. Analysis and interpretation of data: Veeravagu, Cole, Azad. Drafting the article: Ratliff, Cole, Azad. Critically revising the article: Ratliff, Veeravagu. Reviewed submitted version of manuscript: Ratliff, Veeravagu. Statistical analysis: Cole. Administrative/technical/material support: Ratliff. Study supervision: Ratliff.

\section{Correspondence}

John Ratliff, Department of Neurosurgery, Stanford University Medical Center, 300 Pasteur Dr., R291 MC 5327, Stanford, CA 94305-5327. email: jratliff@stanford.edu. 


\section{Appendix}

SUPPLEMENTAL TABLE 1. ICD-9 and CPT codes used to define the procedures and complications in the study cohort

\begin{tabular}{|c|c|}
\hline Procedure/Complication & Code \\
\hline Single-level ACDF (CPT) & 22554 \\
\hline Multilevel ACDF (CPT) & 22585,63076 \\
\hline Ant instrumentation (CPT) & $22845,22846,22847,22851$ \\
\hline Ant decompression (CPT) & 63075 \\
\hline Ant instrumentation (CPT) & $22845,22846,22847,22851$ \\
\hline Pst cervical procedures (CPT) & $\begin{array}{l}63001,63015,63050,63051,63020,63045,63040,63035,63048 \\
\quad 63043,22210,22220,22216,22226,22326,22590,22595,22600 \\
22614,63250,63265,63270,63275,63280,63285\end{array}$ \\
\hline Cervical corpectomy (CPT) & 63081,63082 \\
\hline Ant thoracolumbar decompression (CPT) & $\begin{array}{l}63077,63085,63087,63090,63101,63102,63301,63302,63303 \\
\quad 63305,63306,63307,22857,22862,22556,22558\end{array}$ \\
\hline Pst thoracolumbar decompression (CPT) & $63030,63012,63046,63047,63042,63055,63056,63064$ \\
\hline Pst thoracolumbar fusion (CPT) & 22612,22630 \\
\hline Pst instrumentation (CPT) & $63290,22840,22848,22849,22841,22842,22843,22844$ \\
\hline Pst lumbar discectomy (CPT) & 63030,63042 \\
\hline Nervous system complication, unspecified (ICD-9) & 997.00 \\
\hline CNS complication (ICD-9) & 997.01 \\
\hline Nervous system complication, stroke (ICD-9) & 997.02 \\
\hline Other nervous system complication (ICD-9) & 997.09 \\
\hline Postop infection (ICD-9) & 998.5 \\
\hline Wound dehiscence (ICD-9) & 998.3 \\
\hline Wound hematoma (ICD-9) & 998.1 \\
\hline Wound complication (ICD-9) & $998.3,998.81,998.83,998.4$ \\
\hline Wound complication (CPT) & $10140,10160,10180,12020,12021,20005,21501,22010,22015$ \\
\hline Delirium (ICD-9) & 293 \\
\hline Chronic pain (ICD-9) & $338.2,338.4$ \\
\hline Pulmonary complication (ICD-9) & $997.3,518.4,518.5,518.7,518.81,518.82,518.83,518.84,518.89,519.1$ \\
\hline Neurological complication (ICD-9) & $997.0,430,431,432,433,434,435,436,438.2,438.3,438.4,438.5$ \\
\hline PE (ICD-9) & 415.1 \\
\hline DVT (ICD-9) & $453.4,451,453.8,453.2,453.1,453.9$ \\
\hline Dysrhythmia (ICD-9) & $427,426.1,426,426.3,426.4,426.5,426.6,426.7,426.8$ \\
\hline Dysphagia (ICD-9) & 787.2 \\
\hline
\end{tabular}

